\title{
Uma Abordagem para Condução de Iniciativas de Melhoria de Processos de Software
}

\author{
Mariano Angel Montoni, Cristina Cerdeiral, David Zanetti, \\ Ana Regina Cavalcanti da Rocha
}

\author{
COPPE/UFRJ - Universidade Federal do Rio de Janeiro \\ Caixa Postal 68511 - CEP 21945-970 - Rio de Janeiro - RJ - Brasil \\ \{mmontoni, cerdeiral, zanetti, darocha\}@cos.ufrj.br
}

\begin{abstract}
The success of software process improvement implementation initiatives depends fundamentally on the strategies adopted to support such initiatives. Therefore, it is essential to define adequate implementation strategies to support the achievement of organization objectives considering its specific characteristics. The objective of this work is to present an approach to support the conduction of software process improvement initiatives based on critical success factors identified by a national research with organizations that conducted software process improvement initiatives. This work also presents the functionalities of a set of tools integrated in a process-centered knowledge management environment customized to support the presented approach.
\end{abstract}

Resumo. O sucesso das iniciativas de melhoria de processo de software depende fundamentalmente das estratégias adotadas para apoiar a condução dessas iniciativas. Portanto, é importante definir estratégias de implementação adequadas que atendam os objetivos de negócio da organização e considerem suas características específicas para aumentar os benefícios alcançados com as melhorias implantadas. O objetivo deste trabalho é apresentar uma abordagem para condução de iniciativas de melhoria de processos de software que leva em consideração fatores críticos de sucesso identificados a partir de uma pesquisa nacional com organizações que conduziram iniciativas de melhoria de processo de software. Este trabalho também apresenta as funcionalidades de um conjunto de ferramentas integradas em um ambiente de gerência de conhecimento centrado em processo customizado para apoiar a abordagem proposta.

\section{Introdução}

Aumentar as vantagens competitivas de organizações desenvolvedoras de software é crucial para garantir a sobrevivência destas no mercado. Para aumentar a capacidade das organizações no desenvolvimento de software, diversas abordagens podem ser adotadas, sendo a implementação de melhoria de processo de software a mais amplamente utilizada.

Desenvolvimento de software é uma atividade complexa e processos de software dependem fortemente do comprometimento humano para sua implementação (COLEMAN e O'CONNOR, 2006). Aspectos de comportamento individual e organizacional, geralmente, têm grande influência no sucesso de iniciativas de melhoria (BADDOO e HALL, 2002a; BADDOO e HALL, 2003). Equipes motivadas e satisfeitas 
tendem a implementar melhorias mais eficientemente e os benefícios são prontamente observados. No entanto, quando o contrário ocorre, resistências a mudanças podem se tornar uma barreira crítica na implementação de melhorias nos processos.

A implementação de melhorias em processos envolve atividades intensas em conhecimento. Isto significa que os envolvidos nas iniciativas de melhoria devem possuir profundo conhecimento sobre engenharia de software e serem capazes de usar esse conhecimento para orientar a implementação de melhorias nos processos da organização aumentando as chances de alcançar os resultados esperados (NIAZI et al., 2006). Ações de melhoria de processos requerem também, geralmente, uma quantidade significativa de recursos financeiros para serem colocadas em prática. Além disso, certos benefícios obtidos com as melhorias implementadas não são facilmente mensuráveis, diminuindo a visibilidade do retorno do investimento em melhoria dos processos (GOLDENSON e HERBSLEB, 1995).

Segundo ZAHARAN (ZAHARAN, 1998), a falta de adequação das abordagens adotadas para apoiar a implementação de melhorias nos processos é uma das razões mais comuns para o fracasso das iniciativas de melhoria. A maioria das abordagens existentes apóiam apenas na identificação de "quais" atividades devem ser implementadas, e não o "como" implementar essas atividades (WU et al., 2004; NIAZI et al., 2005).

Considerando que organizações de consultoria em melhoria de processos de software têm a implementação de melhoria de processos como seu negócio principal, e que o seu sucesso implica diretamente no sucesso das iniciativas de melhoria de organizações de software que contratam seus serviços, é fundamental fornecer mecanismos adequados para apoiar organizações de consultoria em melhoria de processos na gerência das iniciativas de implementação de melhoria.

Este trabalho apresenta uma abordagem para condução de iniciativas de melhoria de processos de software. Para apoiar a abordagem, foram definidas ferramentas integradas em um ambiente de gerência de conhecimento centrado em processo com o propósito de permitir que organizações de consultoria em melhoria de processos de software estabeleçam estratégias adequadas para conduzir iniciativas de melhoria de processos de software, bem como permitir a execução e acompanhamento dessas estratégias, além de gerenciar o conhecimento necessário para conduzir iniciativas de melhoria de processo de software. Para possibilitar o desenvolvimento da abordagem, foi desenvolvida uma metodologia para identificação de fatores críticos de sucesso que influenciam iniciativas de melhoria de processo de software. A metodologia e os resultados de sua aplicação são também descritos neste trabalho.

$\mathrm{Na}$ próxima seção são discutidas questões sobre gerência de iniciativas de implementação de melhoria de processo de software. A Seção 3 apresenta uma metodologia para apoiar a identificação de fatores críticos de sucesso que influenciam iniciativas de melhoria. A Seção 4 apresenta a abordagem desenvolvida para condução de iniciativas de melhoria de processo de software, bem como as funcionalidades do apoio ferramental à abordagem. A Seção 5 apresenta algumas considerações finais do trabalho e aponta os próximos passos. 


\section{Gerência de Iniciativas de Implementação de Melhoria de Processo de Software}

Um aspecto importante de ser considerado na gerência de iniciativas de implementação de melhoria de processo de software são as questões relevantes que influenciam o sucesso dessas iniciativas. Essas questões geralmente estão entranhadas na organização de tal forma que é difícil reconhecer tanto sua existência quanto sua importância (ZAHARAN, 1998). Por exemplo, a implementação de melhoria de processo de software envolve a introdução de práticas inovadoras na organização que requerem de seus colaboradores conhecimento aprofundado sobre as novas tecnologias. No entanto, é difícil para a organização reconhecer que seus colaboradores nem sempre possuem as competências necessárias para implementar essas tecnologias e melhorias de forma eficaz e eficiente. Essa possível deficiência é um risco para a implementação da melhoria (pessoas podem achar que a tecnologia ou melhoria não é adequada, quando de fato falta o conhecimento para obter dela o máximo de benefícios).

As questões que exercem influência sobre as iniciativas de implementação de melhoria de processos de software vêm sendo objeto de estudo nas últimas décadas. $\mathrm{O}$ propósito desses estudos é obter um melhor entendimento sobre as questões que influenciam iniciativas de melhoria de processo de software, bem como suas interações, causas, efeitos e formas de tratamento. Essas questões são tratadas comumente por esses estudos como Fatores Críticos de Sucesso (FCS). No entanto, não existe um consenso na área sobre quais são esses fatores nem de que forma eles interagem ou influenciam o sucesso da implementação de melhoria de processo de software.

Segundo NIAZI et al. (NIAZI et al., 2005), as organizações de software, geralmente, não tratam suas iniciativas de melhoria de processos como projetos reais. Dessa forma, torna-se difícil aplicar de forma eficaz e eficiente métodos e técnicas estabelecidos na área de gerência de projetos para apoiar a condução das iniciativas de melhoria. Um outro aspecto importante de ser considerado na implementação de melhoria de processo de software é que as atividades relacionadas às iniciativas de melhoria geralmente envolvem transferência de grande quantidade de conhecimento. Portanto, é importante que responsáveis pelas iniciativas de melhoria de processo de software tenham acesso a esse conhecimento para facilitar a definição, execução e acompanhamento de programas de melhoria.

BADDOO e HALL (BADDOO e HALL, 2002b) sugerem que o aprimoramento do conhecimento sobre a relação entre os motivadores da melhoria de processo de software para diferentes grupos de engenheiros de software permitirá que gerentes de melhoria implementem iniciativas de melhoria mais eficientemente. EL-EMAM et al. (EL-EMAM et al., 2001) indicam também que diferenças culturais das organizações afetam diretamente o sucesso da melhoria de processo de software.

Alguns trabalhos foram desenvolvidos para apoiar a gerência de implementação de melhoria de processos de software. NIAZI et al. (NIAZI et al., 2005) desenvolveram um framework de implementação de melhoria de processo de software chamado de SPIIF a partir de estudos experimentais sobre os fatores críticos de sucesso (FCS) para melhoria de processo de software. Estes estudos tiveram dois focos principais: descobrir o "que" é crítico na implementação de melhorias de processo de software e descobrir o "como" implementar melhorias nas organizações. WILSON et al. (WILSON et al., 2001) desenvolveram um framework para avaliação de sucesso de iniciativas de 
melhoria de processo de software e validaram essa abordagem com entrevistas em grupos em sete organizações do Reino Unido. DYB (DYBA, 2000) desenvolveu um instrumento para medição de fatores críticos de sucesso na melhoria de processo de software baseados em dados coletados a partir de 120 organizações de software.

\section{Uma Metodologia para Identificação de Fatores Críticos de Sucesso que Influenciam Iniciativas de Melhoria de Processo de Software}

Para apoiar a condução de estudos experimentais na área de melhoria de processos de software, foi desenvolvida uma metodologia que combina técnicas qualitativa e quantitativa para coletar e analisar dados relacionados a fatores que têm impacto positivo ou negativo em experiências de iniciativas de melhoria de processo de software de instituições implementadoras de melhoria de processo. Os métodos e técnicas utilizados neste estudo foram largamente aplicados por outros estudos com os mesmos objetivos de pesquisa, mas com foco em outros contextos. A metodologia desenvolvida será apresentada por meio da descrição dos passos realizados na sua aplicação em um estudo específico (MONTONI, 2007; MONTONI e ROCHA, 2007).

Inicialmente, foram definidos um contexto e escopo para o estudo. $\mathrm{O}$ estudo foi restringido para analisar apenas experiências de um grupo específico de implementadores de melhoria de processo de software que participaram em iniciativas de melhoria baseadas em modelos e padrões de referência (por exemplo, CMMI e MPS.BR). Neste estudo, teve-se uma preocupação de identificar fatores críticos de sucesso sob dois pontos de vista. O primeiro ponto de vista é o de implementadores que participaram como consultores em projetos de implementação de melhoria de processo de software. O segundo ponto de vista é o de membros das organizações envolvidas nos projetos de melhoria.

Neste estudo, foi aplicado inicialmente o método Grounded Theory (GT) (STRAUSS e CORBIN, 1998). O primeiro passo para aplicação desse método foi coletar os dados por meio da aplicação de dois tipos de questionários com o objetivo de identificar fatores que influenciam a implementação de melhoria de processo de software. O primeiro tipo de questionário foi enviado para um grupo selecionado de implementadores de melhoria de processo de software que participaram como consultores em organizações nacionais de diversos tipos. $O$ segundo tipo de questionário foi enviado para membros de organizações envolvidos em projetos de implementação de melhoria. Os questionários não continham nenhum item prédeterminado e os participantes o preencheram separadamente. No total, 25 questionários foram retornados contendo descrições gerais sobre fatores que influenciaram as iniciativas de implementação de melhoria de processo de software. Depois de retornados os questionários, foram associados códigos a cada uma das declarações nos questionários representando tipos de achados de fatores críticos de sucesso que influenciaram iniciativas de implementação de melhoria de processo de software. No total, 66 códigos diferentes foram identificados por meio da análise dos questionários.

Os dados codificados (achados) foram, então, analisados e categorizados. Essas categorias foram denominadas de propriedade de fator crítico de sucesso. A associação entre os achados e uma propriedade de fator crítico de sucesso foi classificada como um achado representando a presença ou a ausência de um fator crítico de sucesso em um contexto específico da análise. A teoria começa a tomar forma à medida que mais 
relações são identificadas entre os achados e as propriedades de fator crítico de sucesso. Essa teoria foi refinada e mais conhecimento sobre os fatores críticos de sucesso foram integrados até que a coleta e análise de mais dados não agregavam nenhum novo conhecimento à teoria. Como resultado desse processo de refinamento e integração, chegou-se à categoria central denominada "Fatores que facilitam e dificultam a condução de iniciativas de melhoria de processos de software". Esse conhecimento sobre fatores críticos de sucesso, suas propriedades e relacionamentos formam o que STRAUSS e CORBIN (STRAUSS e CORBIN, 1998) chamam de framework teórico.

As técnicas de análise estatística MDS (STATSOFT, 2004) e PCA (KIM e MUELLER, 1978) foram aplicadas para derivar e agregar os fatores críticos de sucesso principais. O primeiro passo para executar a análise de MDS foi estabelecer um dicionário de conteúdo categorizado de todas as propriedades dos fatores críticos de sucesso. Em seguida, uma matriz de dados foi criada com base em quantas ocorrências das propriedades de fatores críticos de sucesso foram identificadas por cada um dos participantes do estudo. Essa matriz foi utilizada para calcular correlações multivariadas entre as propriedades dos fatores críticos de sucesso. Finalmente, essas correlações foram utilizadas para desenhar as distâncias geométricas entre os fatores críticos de sucesso no Gráfico MDS.

A técnica PCA foi aplicada na matriz de dados de propriedades de fatores críticos de sucesso com o objetivo de identificar as propriedades com relacionamento estatisticamente significativo. Por meio da aplicação da técnica de análise PCA, as propriedades receberam um valor de carga final para cada um dos componentes (grupos) de fatores críticos de sucesso extraído. Neste estudo, foi considerado o valor 0,55 como o valor de corte dado o tamanho limitado das fontes de dados analisadas.

A confiabilidade de relações entre variáveis e componentes extraídos utilizando a técnica de PCA pode ser realizada por meio do cálculo do coeficiente alpha de Cronbach (CRONBACH, 1951). Esta é uma medida comumente utilizada para avaliar a confiabilidade de escalas de medição subjetivas. O coeficiente alpha de Cronbach pode variar de 0 a 1 , onde 1 é a confiabilidade perfeita e 0 é a máxima falta de confiabilidade. Nunnally sugere que para os estágios iniciais de uma pesquisa, um valor de coeficiente alpha de Cronbach próximo de 0,7 é aceitável (NUNNALLY, 1978). Nove componentes de fatores críticos de sucesso foram analisados utilizando o coeficiente alpha de Cronbach. Aproximadamente $85 \%$ da variação é explicada por esses nove componentes. Esse é um valor muito bom considerando a natureza exploratória deste estudo. Os fatores extraídos foram utilizados para construir componentes de fatores críticos de sucesso.

O fator 1 possui um coeficiente alpha de Cronbach de valor 0,81 demonstrando que esse componente de fatores críticos de sucesso possui uma confiabilidade muito boa. Apesar do fator 2 ter poucas variáveis para calcular o coeficiente alpha de Cronbach, os altos valores de carga de rotação da análise de fator exploratória para esse componente indicam uma alta correlação entre as variáveis do fator 2 . $\mathrm{O}$ fator 3 possui um coeficiente alpha de Cronbach de valor 0,56. Apesar deste número não ser muito alto, os altos valores de carga de rotação também indicam uma alta correlação entre as variáveis do fator 3. O fator 4 possui um coeficiente alpha de Cronbach de valor 0,81 demonstrando que esse componente de fatores críticos de sucesso tem uma 
confiabilidade muito boa. $\mathrm{O}$ fator 5 tem o coeficiente alpha de Cronbach de valor 0,58. Apesar desse número não ser muito alto, é relativamente bom para um fator consistindo de apenas 3 variáveis. Esses cinco componentes de fator crítico de sucesso explicam $65 \%$ da variação total o que é um valor muito bom considerando o número reduzido de casos analisados. Os fatores 6 a 9 possuem muito poucas variáveis para calcular o valor do coeficiente alpha de Cronbach e não possuem valores de carga de rotação altos da análise de fator exploratória. Portanto, os fatores 1 a 5 foram considerados os componentes principais de fatores críticos de sucesso.

A Figura 1 apresenta o gráfico desses componentes e as respectivas variáveis como resultado da aplicação da técnica MDS das correlações multivariadas calculadas para as propriedades dos fatores críticos de sucesso. Os componentes principais de fatores críticos de sucesso com confiabilidade estatística identificados por meio da aplicação de PCA são também destacados na Figura 1.

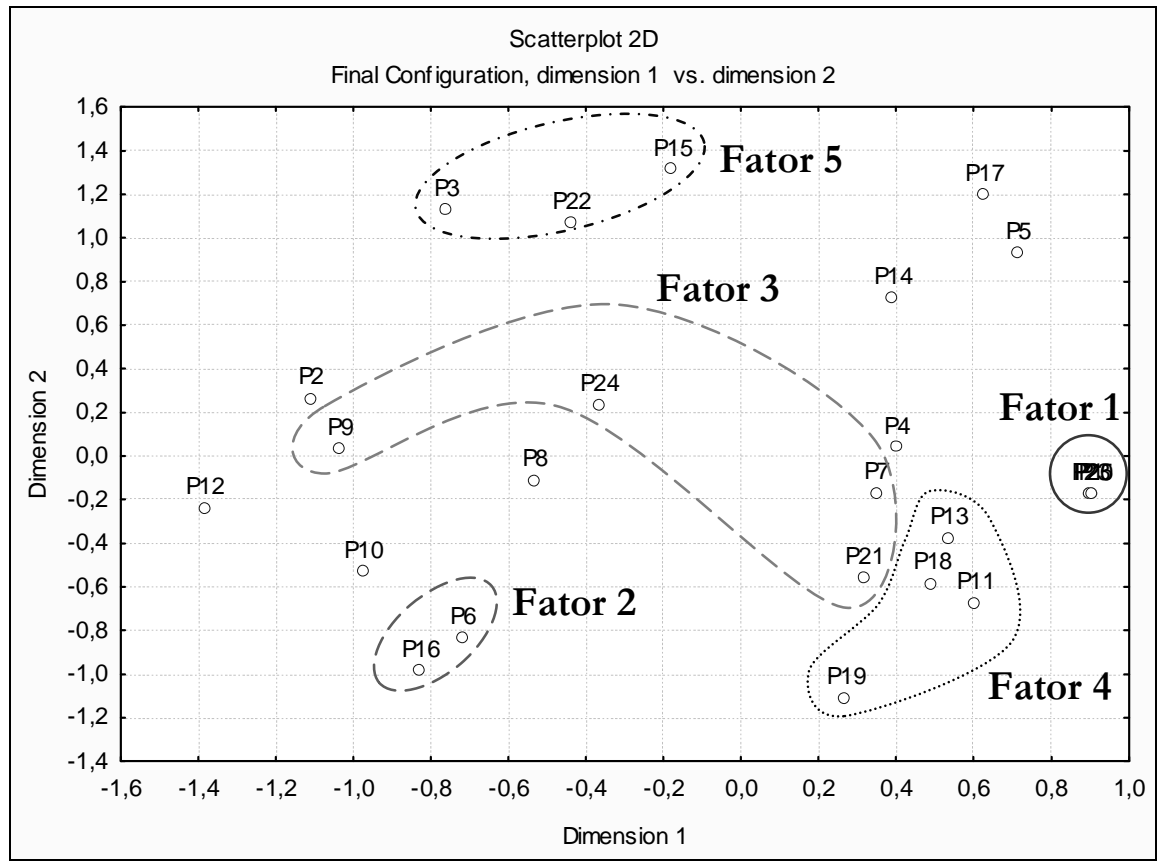

Figura 1. Gráfico dos componentes principais de fatores críticos de sucesso.

A composição dos componentes principais de fatores críticos de sucesso é descrita a seguir. O Fator 1 foi chamado de "Ambiente" e é composto das seguintes variáveis: (i) competências dos membros da organização (Grau de competências em engenharia de software dos membros da organização), (ii) conciliação de interesses (Grau de adequação da conciliação de interesses na implementação de processos), (iii) estrutura da organização (Grau de estabilidade interna na organização), (iv) motivação e satisfação dos membros da organização (Grau de satisfação dos membros da organização), (v) política de reconhecimento à colaboração na melhoria dos processos (Existência de política de reconhecimento à colaboração na melhoria dos processos) e (vi) respeito da consultoria pelos membros da organização (Grau de relacionamento dos membros da organização com a consultoria especializada). Todas estas variáveis medem a capacidade ambiental para estabelecer e manter iniciativas de melhoria de processo de software. Essas variáveis medem se existem condições favoráveis para 
iniciar e manter uma iniciativa de melhoria sob dois pontos de vista: o indivíduo e a organização. As medidas do indivíduo estão relacionadas à satisfação dos membros da organização e ao relacionamento entre esses membros e a equipe de consultoria de melhoria de processo de software. As medidas da organização são relacionadas à conciliação entre os objetivos estratégicos e interesses da organização na melhoria de processo de software, e à estabilidade organizacional interna.

O Fator 2 foi chamado de "Estratégia" e é composto das seguintes variáveis: (i) conscientização dos benefícios da implementação da melhoria dos processos (Grau de conscientização dos membros da organização quanto aos benefícios obtidos com a implantação dos processos) e (ii) estratégia de implementação de melhoria de processo de software (Grau de adequação da gerência do projeto de implementação da melhoria dos processos). Este fator indica que uma estratégia de melhoria de processo de software deve garantir que os membros da organização têm consciência dos benefícios que podem ser alcançados com a implementação da melhoria de processo de software.

O Fator 3 foi chamado de "Institucionalização" e é composto das seguintes propriedades: (i) estratégia de implementação de melhoria de processo de software (Grau de adequação da relação push-pull na implementação dos processos), (ii) estrutura da organização (Grau de rotatividade de pessoal) e (iii) processos (Grau de adequação dos processos/procedimentos e Grau de institucionalização das melhorias implementadas nos projetos). Estas variáveis medem o grau de institucionalização das melhorias pela caracterização do grau de resistência da institucionalização dos processos e procedimentos a mudanças estruturais da organização, por exemplo, rotatividade de pessoal, e a dificuldades inerentes da implementação de melhoria de processo de software nos diferentes níveis organizacionais.

O Fator 4 foi chamado de "Comprometimento" e é composto das seguintes propriedades: (i) apoio, comprometimento e envolvimento (Grau de apoio, comprometimento e envolvimento da alta gerência), (ii) competências dos membros da organização (Grau de competências em engenharia de software dos membros da organização) e (iii) recursos (Grau de disponibilidade de recursos financeiros dos membros da organização para atividades de melhoria de processo e Grau de disponibilidade de tempo dos membros da organização para atividades de melhoria de processo). Todas estas variáveis são consideradas indicadores de comprometimento para a melhoria de processo de software. Uma alta gerência comprometida para a melhoria de processo de software fornece recursos financeiros adequados desde a concepção do programa de melhoria e ao longo dos projetos de melhoria. Além do mais, uma gerência sênior comprometida garante que os membros da organização têm competências adequadas e tempo disponível para executar mudanças de processos eficientemente.

O Fator 5 foi chamado de "Motivação e aceitação" e é composto das seguintes propriedades: (i) aceitação a mudanças (Grau de aceitação a mudanças), (ii) motivação e satisfação dos membros da organização (Grau de motivação dos membros da organização) e (iii) respeito da consultoria pelos membros da organização (Grau de confiança dos membros da organização na consultoria especializada). Este fator indica que a equipe de melhoria de processo de software é um facilitador da aceitação dos membros da organização para a institucionalização de mudanças nos processos promovidas pelas iniciativas de melhoria de processo de software. 


\section{Uma Abordagem para Condução de Iniciativas de Melhoria de Processos de Software}

Para apoiar organizações na gerência de seus programas de melhoria de processo de software de forma a tratar adequadamente questões que influenciam o sucesso desses programas, foi definida uma abordagem apoiada por uma infra-estrutura computacional baseada em um ambiente customizado de gerência de conhecimento centrado em processo.

\subsection{Arquitetura da Abordagem}

A arquitetura da abordagem proposta é composta de diferentes componentes com objetivos e responsabilidades bem definidos, como pode ser observado na Figura 2 e explicado a seguir.

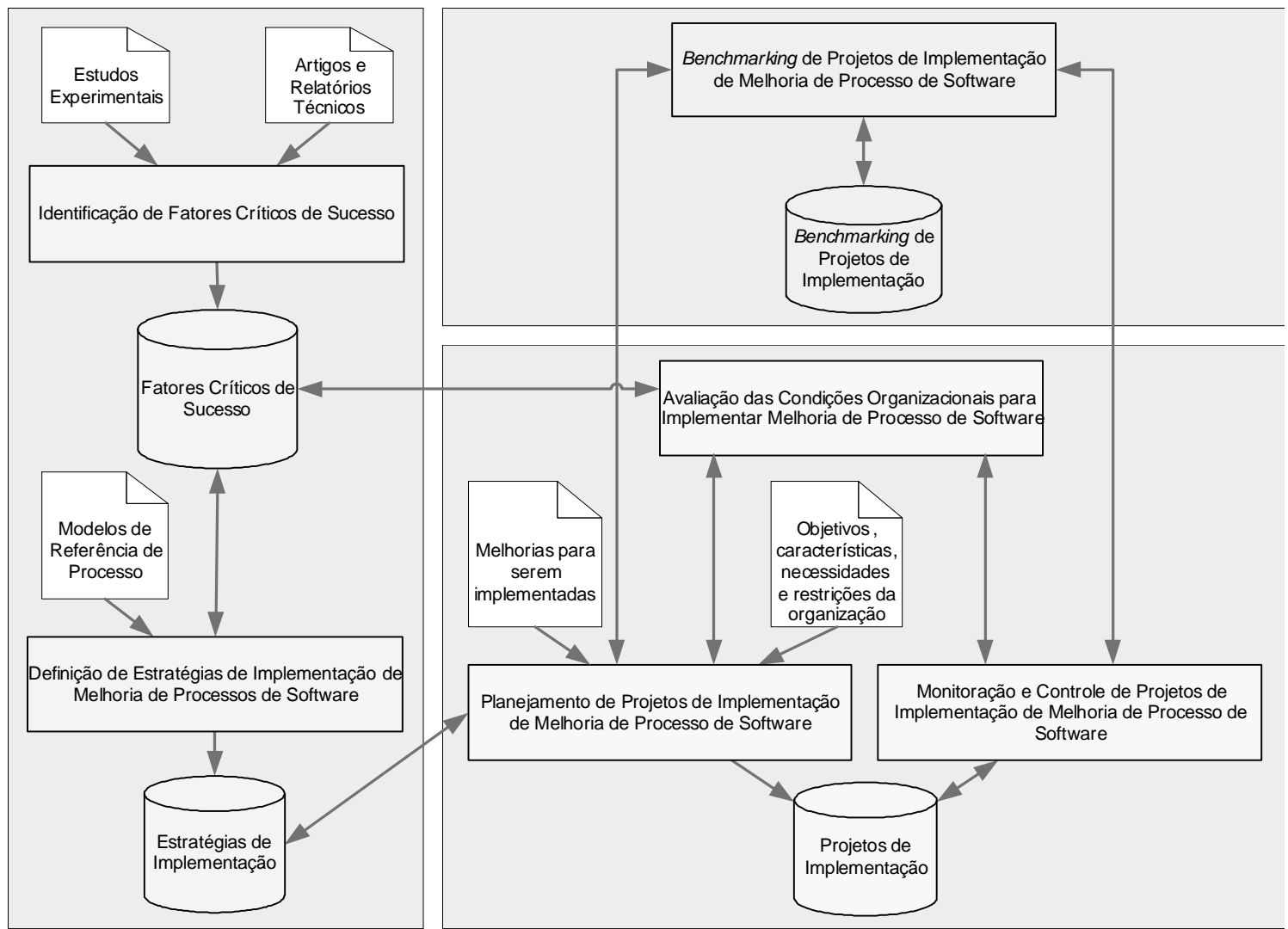

Figura 2. Componentes da Abordagem para Condução de Iniciativas de Melhoria de Processos de Software (MONTONI, 2007).

O primeiro grupo de componentes aborda os conhecimentos sobre (i) os fatores críticos de sucesso que influenciam o resultados das iniciativas de melhoria e (ii) as estratégias de condução de implementação de melhorias de processos de software. Os componentes deste grupo são:

- Identificação de Fatores Críticos de Sucesso: este componente apóia a aquisição de conhecimento sobre os fatores críticos de sucesso que influenciam iniciativas de melhoria. O componente deve capturar conhecimento de diversas fontes, 
como artigos, relatórios técnicos e estudos experimentais e auxiliar a aplicação da metodologia de identificação de fatores críticos de sucesso apresentada na Seção 3 deste trabalho.

- Definição de Estratégias de Implementação de Melhoria de Processos de Software: este componente apóia a aquisição de conhecimento sobre as estratégias de implementação de melhorias de processos de software em contextos específicos, considerando as características encontradas nas organizações que favorecem ou dificultam as iniciativas de melhoria. O componente deve permitir que diferentes instituições de consultoria armazenem (i) suas estratégias definidas com base em diferentes abordagens e modelos de referência de melhoria de processos, (ii) o contexto no qual estas estratégias são aplicáveis e (iii) diretrizes para adaptação das estratégias para apoiar a condução de iniciativas específicas de melhoria. Além disso, o componente deve permitir que uma organização de consultoria em melhoria de processos mantenha as suas estratégias de implementação com base no conhecimento adquirido a partir da condução de iniciativas anteriores de melhoria.

O segundo grupo de componentes aborda a aplicação de técnicas de benchmarking em projetos de melhoria de processos de software. Os componentes deste grupo são:

- Benchmarking de Projetos de Implementação de Melhoria de Processo de Software: este componente apóia a identificação de melhores práticas de uma instituição de consultoria em melhoria de processos e a aplicação destas práticas em novas iniciativas de melhoria. Além disso, o componente mantém o conhecimento do desempenho dos projetos de melhoria de processos de software e os contextos encontrados nas organizações favoráveis ou não às iniciativas de melhoria, auxiliando as instituições de consultoria em implementação de melhoria de processos de software a determinarem o desempenho esperado em projetos com contextos similares atuais e futuros e a avaliarem o desempenho de seus projetos atuais com contextos similares.

O terceiro grupo de componentes aborda a gerência e avaliação dos projetos de implementação de melhoria de processos de software. Os componentes deste grupo são:

- Planejamento de Projetos de Implementação de Melhoria de Processo de Software: este componente apóia o planejamento de projetos de implementação de melhoria de processos de software, auxiliando a seleção e adaptação de estratégias de implementação com base nas características encontradas nas organizações que favorecem ou dificultam as iniciativas de melhoria.

- Monitoração e Controle de Projetos de Implementação de Melhoria de Processo de Software: este componente apóia a monitoração das atividades executadas no contexto dos projetos de implementação de melhoria de processos de software, através de dados quantitativos e qualitativos, permitindo a identificação do desempenho dos projetos de melhoria. O componente deve apoiar também a monitoração e o tratamento de forma contínua dos fatores que podem influenciar significativamente o resultado das iniciativas. 
- Avaliação das Condições Organizacionais para Implementar Melhoria de Processo de Software: este componente apóia a identificação dos fatores que podem influenciar o sucesso de iniciativas de melhoria com base nas características encontradas nas organizações, no início da iniciativa de melhoria e durante a sua condução.

\subsection{Os Conceitos de Processo Padrão e Estratégia de Implementação de Projetos de Melhoria de Processos de Software}

Dois conceitos utilizados neste trabalho são fundamentais e necessitam ser explicados. O primeiro conceito é o de processo padrão de melhoria de processos de software. Uma organização de consultoria em implementação de melhoria de processos de software pode possuir um ou mais processos padrão de melhoria, no qual as fases e subprocessos são definidos em alto nível. Os processos padrão de melhoria podem ser construídos com base em abordagens de implementação de melhoria de processos de software. Um exemplo seria um processo padrão definido com as fases e subprocessos encontrados no IDEAL (GREMBA e MYERS, 1997) ou no SPI-KM (SANTOS et al., 2007a).

O segundo conceito é o de estratégia de implementação de melhoria de processos de software. Uma instituição de consultoria em implementação de melhoria de processos de software pode possuir uma ou mais estratégias de implementação de melhorias aplicáveis a cada um dos subprocessos de um ou mais processos padrão de melhoria de processos de software. As estratégias especializam e adaptam um subprocesso do processo padrão de melhorias, definindo as atividades a serem realizadas em cada subprocesso e gerando o processo definido para o projeto de melhoria.

Uma estratégia de implementação de melhoria de processos de software aplicada a um subprocesso do processo padrão de melhoria define: (i) as atividades realizadas no subprocesso e suas descrições, como os artefatos requeridos e produzidos pelas atividades e os perfis normalmente responsáveis por suas execuções; (ii) os contextos encontrados nas organizações nos quais a estratégia pode ser aplicada; (iii) os conhecimentos necessários para a realização das atividades definidas pela estratégia; (iv) os recursos necessários para a realização das atividades definidas pela estratégia; (v) os riscos associados com a estratégia selecionada e observados em projetos anteriores; (vi) as ações de mitigação e contingência relacionadas com os riscos associados com a estratégia selecionada e os resultados obtidos nas suas execuções em projetos anteriores; (vii) o tempo e esforço normalmente demandados pelas atividades definidas pela estratégia, com base nas suas execuções em projetos anteriores; e (viii) a comunicação necessária para o sucesso das atividades definidas pela estratégia, baseada nas suas execuções em projetos anteriores.

Como exemplo de estratégia, podemos pensar no subprocesso "Estabelecer a Infra-estrutura" definido na abordagem de implementação de melhoria de processos de software IDEAL (GREMBA e MYERS, 1997), na fase Iniciar. Dependendo do contexto encontrado nas organizações, as instituições de consultoria em implementação de melhoria de processos de software podem optar por fornecer ou indicar infra-estruturas diferentes. Caso as organizações não possuam o apoio ferramental necessário para alcançar as melhorias desejadas, por exemplo, as instituições de consultoria podem 
sugerir várias ferramentas, de acordo com o contexto encontrado nas organizações, como facilidade de aprendizado, ferramentas já utilizadas pelas organizações e necessidades demandadas pelas melhorias almejadas. Dependendo do apoio ferramental sugerido, as instituições de consultoria em implementação de melhoria de processos de software podem realizar atividades específicas do apoio sugerido. Um exemplo é a utilização da Estação TABA (FERREIRA et al., 2006; MONTONI et al., 2006; MONTONI et al., 2007) fornecida pela COPPE/UFRJ para as organizações que a contratam para consultoria em processos de software. A COPPE/UFRJ precisa realizar customizações na Estação TABA para adaptá-la ao contexto das organizações que a utilizam, realizando atividades relacionadas com estas adaptações, como o cadastro do processo padrão de desenvolvimento das organizações na ferramenta.

\subsection{Apoio Ferramental}

Um conjunto de ferramentas está sendo desenvolvido para apoiar a aplicação da abordagem apresentada acima. Essas ferramentas estão sendo integradas em um ambiente customizável de gerência de conhecimento centrado em processo chamado de CORE-KM (Customizable Organizational Resources Environment with Knowledge Management) (GALOTTA et al., 2004).

Um ambiente de gerência de conhecimento foi customizado a partir do COREKM para a COPPE/UFRJ considerando suas características particulares e processos organizacionais. Esse ambiente está sendo utilizado para apoiar a aplicação da estratégia de implementação de melhoria de processo da COPPE/UFRJ, também chamada de SPIKM, em organizações desenvolvedoras de software. A estratégia SPI-KM foi utilizada para coordenar inúmeras iniciativas de implementação de melhoria de processos de software em diversas organizações desenvolvedoras de software do Brasil (SANTOS et al., 2007a; SANTOS et al., 2007b). A realização de avaliações formais nessas organizações indicam que a estratégia da COPPE/UFRJ é eficaz e eficiente. No entanto, a aplicação da estratégia foi realizada sem nenhum apoio computacional, o que criou inúmeras dificuldades, por exemplo, a dificuldade de transferir o conhecimento sobre a estratégia para consultores em melhoria iniciantes.

O conjunto de ferramentas definidas para apoiar a abordagem é o seguinte:

- Ferramenta de apoio à Gerência de Estratégia de Implementação de Melhoria de Processo: o objetivo desta ferramenta é fornecer apoio para (i) gerenciar informações sobre fatores críticos de sucesso que influenciam iniciativas de melhoria de processo de software e (ii) gerenciar informações de estratégias de implementação de melhoria de processo de software de uma organização de consultoria específica.

- Ferramenta de apoio à Benchmarking de Projetos de Implementação de Melhoria de Processo de Software: o objetivo desta ferramenta é fornecer apoio para (i) definir uma base de benchmarking de projetos de implementação de melhoria de processo de software coordenados por uma organização de consultoria específica, (ii) fornecer mecanismos de gerência de informações de projetos de implementação de melhoria de processo de software coordenados por uma organização de consultoria específica e (iii) fornecer mecanismos de consulta de informações de projetos similares de implementação de melhoria de processo de 
software coordenados por uma organização de consultoria específica. A Figura 3 apresenta a tela desta ferramenta que consolida informações sobre o desempenho de diferentes estratégias de implementação de melhoria de processo de software.

- Ferramenta de apoio à Gerência de Projetos de Implementação de Melhoria de Processo de Software: o objetivo desta ferramenta é fornecer apoio para (i) planejar projetos de implementação de melhoria de processo de software coordenados por uma organização de consultoria específica, (ii) monitorar e controlar projetos de implementação de melhoria de processo de software coordenados por uma organização de consultoria específica e (iii) avaliar as condições organizacionais para implementar melhoria de processo de software. A Figura 4 apresenta a tela desta ferramenta que apóia a identificação de fatores críticos de sucesso que influenciam projetos de implementação de melhoria de processo de software. A partir desta tela, o gerente do projeto de implementação pode identificar fatores críticos de sucesso presentes em um projeto específico e definir um plano de gerência de risco constituído de ações de mitigação e contingenciamento para gerenciar os fatores avaliados negativamente como ameaças potenciais ao sucesso do projeto.

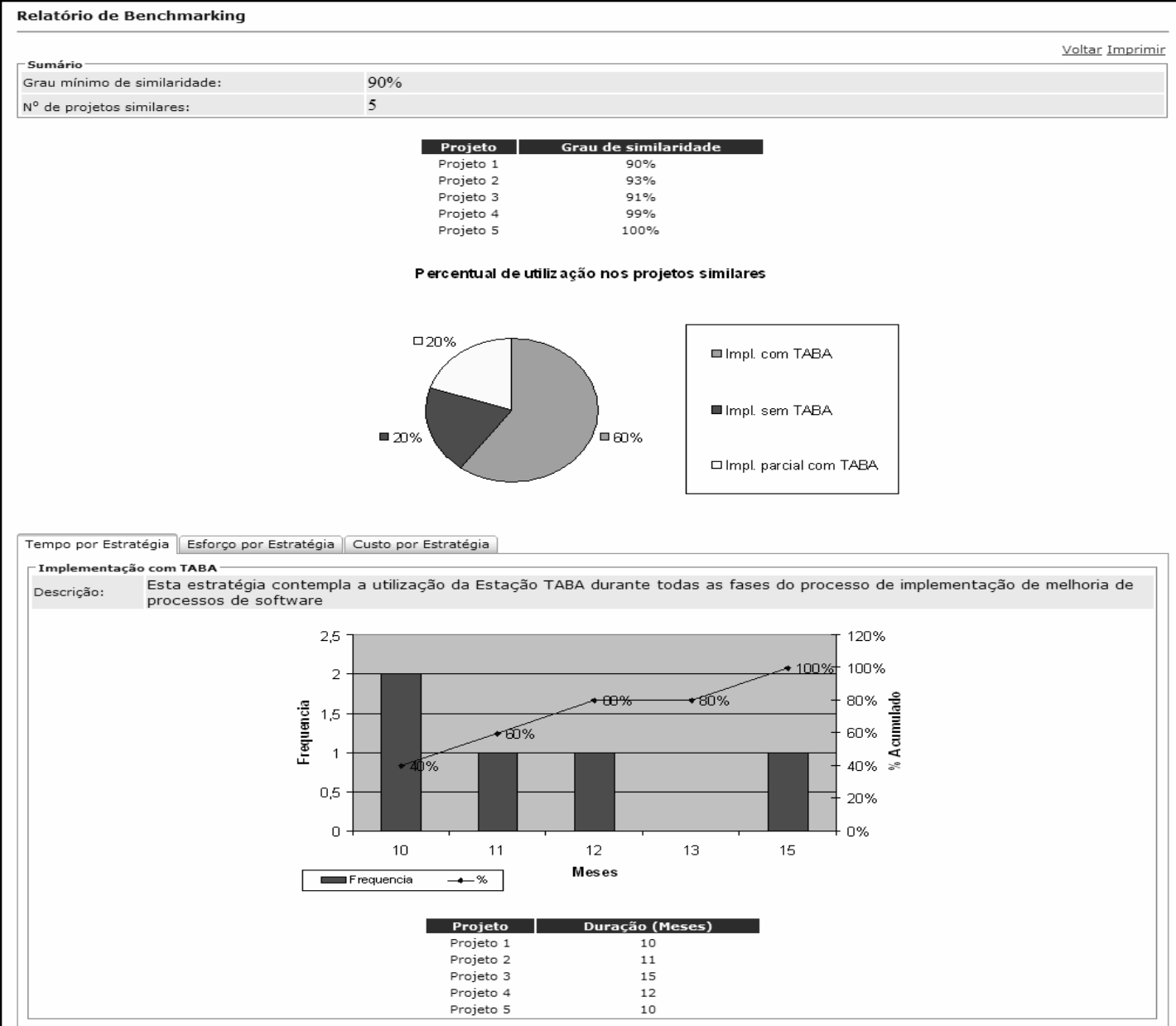

Figura 3. Tela do relatório de Benchmarking. 


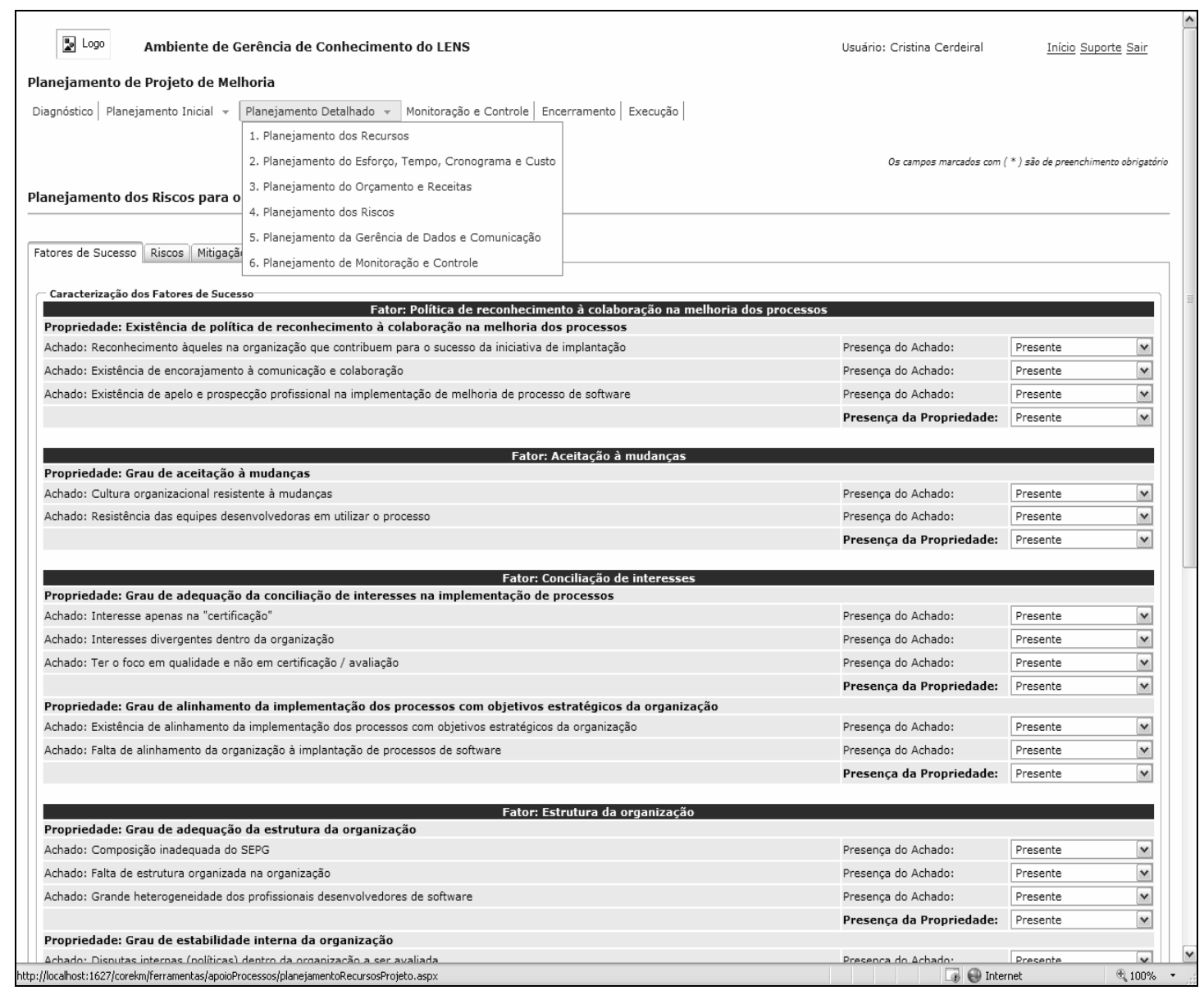

Figura 4. Tela de identificação de fatores críticos de sucesso.

\section{Conclusão}

Este trabalho apresentou uma abordagem para condução de iniciativas de melhoria de processo de software. Também foi apresentada uma metodologia para apoiar a identificação de fatores críticos de sucesso que influenciam iniciativas de melhoria de processo de software. O apoio ferramental desenvolvido para apoiar a abordagem proposta foi também discutido.

Um estudo de casos de aplicação da abordagem apresentada está sendo planejado para avaliar a abordagem e identificar seus pontos fortes, pontos fracos e oportunidades de melhoria. A partir deste estudo, espera-se que seja verificado, em organizações de consultoria em melhoria de processo de software, os seguintes benefícios principais com o uso da abordagem proposta neste trabalho: (i) acumular conhecimento sobre fatores críticos de sucesso que influenciam iniciativas de melhoria de processos de software; (ii) acumular conhecimento sobre estratégias de implementação de melhoria de processos de software; (iii) facilitar a seleção e adaptação de estratégias de implementação de melhoria de processos de software em contextos específicos de implementação; (iv) facilitar a avaliação das condições organizacionais para implementar melhorias nos processos de software; (v) facilitar a gerência de iniciativas de melhoria de processos de software com base em estratégias de implementação mais eficientes e eficazes; (vi) aumentar a visibilidade dos resultados das iniciativas de melhoria e diminuir os custos, prazo e esforço na condução dessas 
iniciativas; e (vii) preservar o conhecimento relacionado à condução de iniciativas de melhoria e facilitar a identificação de melhores práticas na implementação de melhoria de processos de software.

\section{Referências}

BADDOO, N., HALL, T., 2002a, "Motivators of Software Process Improvement: An analysis of practitioners' views", Journal of Systems and Software, v. 62, n. 2, pp. 85-96.

BADDOO, N., HALL, T., 2002b, "Software process improvement motivators: An analysis using multidimensional scaling", Empirical Software Engineering, v. 7, n. 2, pp. 93-114.

BADDOO, N., HALL, T., 2003, "De-motivators for software process improvement: An analysis of practitioners' views", Journal of Systems and Software, v. 66, n. 1, pp. 23-33.

COLEMAN, G., O'CONNOR, R., 2006, "Software process in practice: A Grounded Theory of the irish software industry", v. 4257 LNCS, pp. 28-39, Joensuu, Finland.

CRONBACH, L.J., 1951, "Coefficient Alpha and the Internal Consistency of Tests", Psychometrica, v. 16 (September), pp. 297-334.

DYBA, T., 2000, "An Instrument for measuring the key factors of success in software process improvement", Empirical Software Engineering, v. 5, n. 4, pp. 357-390.

EL-EMAM, K., GOLDENSON, D., MCCURLEY, J., et al., 2001, "Modelling the likelihood of software process improvement: An exploratory study", Empirical Software Engineering, v. 6, n. 3, pp. 207-229.

FERREIRA, A.I.F., SANTOS, G., CERQUEIRA, R., et al., 2006, "Taba workstation: Supporting software process improvement initiatives based on software standards and maturity models", v. 4257 NCS, pp. 207-218, Joensuu, Finland.

GALOTTA, C., ZANETTI, D., ROCHA, A.R., et al., 2004, "Organizational Learning Based on a Customizable Environment for Knowledge Management Using Intranet". In: E-LEARN 2004 - World Conference on e-Learning in Corporate, Government, Healthcare \& Higher Education, v. 2, pp. 2626-2633, Washington, EUA.

GOLDENSON, D.R., HERBSLEB, J.D., 1995, After the Appraisal: A Systematic Survey of Process Improvement, its Benefits and Factors that Influence Success, CMU/SEI-95-TR-009, Software Engineering Institute.

GREMBA, J., MYERS, C., "The IDEAL Model: A Practical Guide for Improvement ". In: http://www.sei.cmu.edu/ideal/ideal.bridge.html.

KIM, J., MUELLER, C., 1978, Factor Analysis: Statistical Methods and Practical Issues, Sage Publications.

MONTONI, M., 2007, Uma Abordagem para Condução de Iniciativas de Melhoria de Processos de Software, Exame de Qualificação para o Doutorado, COPPE, UFRJ, Rio de Janeiro. 
MONTONI, M., ROCHA, A.R., 2007, "A Methodology for Identifying Critical Success Factors that Influence Software Process Improvement Initiatives: An Application in the Brazilian Software Industry", Lecture Notes in Computer Science (LNCS), LNCS 4764, EuroSPI - European Systems \& Software Process Improvement and Innovation (Setembro), pp. 175-186.

MONTONI, M., SANTOS, G., ROCHA, A.R., et al., 2006, "Taba workstation: Supporting software process deployment based on CMMI and MR-MPS.BR", v. 4034 NCS, pp. 249-262, Amsterdam, Netherlands.

MONTONI, M., SANTOS, G., ROCHA, A.R., et al., 2007, "MPS Model and TABA Workstation: Implementing Software Process Improvement Initiatives in Small Settings". In: Software Quality, 2007. WoSQ'07: ICSE Workshops 2007. Fifth International Workshop on, pp. 4-4.

NIAZI, M., WILSON, D., ZOWGHI, D., 2005, "A framework for assisting the design of effective software process improvement implementation strategies", Journal of Systems and Software, v. 78, n. 2, pp. 204-222.

NIAZI, M., WILSON, D., ZOWGHI, D., 2006, "Critical success factors for software process improvement implementation: An empirical study", Software Process Improvement and Practice, v. 11, n. 2, pp. 193-211.

NUNNALLY, J.C., 1978, Psychometric Theory, 2nd ed. New York, McGraw-Hill.

SANTOS, G., MONTONI, M., FIGUEIREDO, S., et al., 2007a, "SPI-KM Lessons Learned from Appling a Software Process Improvement Strategy Supported by Knowledge Management". In: 8th International PROFES (Product Focused Software Development and Process Improvement), LNCS 4589, pp. 81-95, Riga, Latvia, July.

SANTOS, G., MONTONI, M., VASCONCELLOS, J., et al., 2007b, "Implementing Software Process Improvement Initiatives in Small and Medium-Size Enterprises in Brazil". In: 6th QUATIC (International Conference on the Quality of Information and Communications Technology), Lisboa, Portugal, Setembro.

STATSOFT, 2004, "STATISTICA Electronic Manual", StatSoft Inc.

STRAUSS, A., CORBIN, J.M., 1998, Basics of Qualitative Research: Techniques and Procedures for Developing Grounded Theory, 2nd ed., Sage Publications.

WILSON, D.N., HALL, T., BADDOO, N., 2001, "A framework for evaluation and prediction of software process improvement success", Journal of Systems and Software, v. 59, n. 2, pp. 135-142.

WU, M., YING, J., YU, C., 2004, "A methodology and its support environment for benchmark-based adaptable software process improvement", v. 6, pp. 51835188, The Hague, Netherlands.

ZAHARAN, S., 1998, Software Process Improvement - Practical Guidelines for Business Success, Addison-Wesley. 